\title{
Frontières
}

\section{Jean-Marc Dufort, Euthanasie et aide au suicide, Montréal, Bellarmin, 1996, 100 pages}

\section{Bernard Jobin}

Volume 12, numéro 1, automne 1999

URI : https://id.erudit.org/iderudit/1074524ar

DOI : https://doi.org/10.7202/1074524ar

Aller au sommaire du numéro

Éditeur(s)

Université du Québec à Montréal

ISSN

1180-3479 (imprimé)

1916-0976 (numérique)

Découvrir la revue

Citer ce compte rendu

Jobin, B. (1999). Compte rendu de [Jean-Marc Dufort, Euthanasie et aide au suicide, Montréal, Bellarmin, 1996, 100 pages]. Frontières, 12(1), 116-118.

https://doi.org/10.7202/1074524ar

Ce document est protégé par la loi sur le droit d'auteur. L'utilisation des services d'Érudit (y compris la reproduction) est assujettie à sa politique d'utilisation que vous pouvez consulter en ligne.

https://apropos.erudit.org/fr/usagers/politique-dutilisation/
Cet article est diffusé et préservé par Érudit.

Érudit est un consortium interuniversitaire sans but lucratif composé de l'Université de Montréal, l'Université Laval et l'Université du Québec à Montréal. Il a pour mission la promotion et la valorisation de la recherche. https://www.erudit.org/fr/ 
Jean-Marc Dufort

\section{Euthanasie et aide} au suicide

Montréal, Bellarmin, 1996, 100 pages.

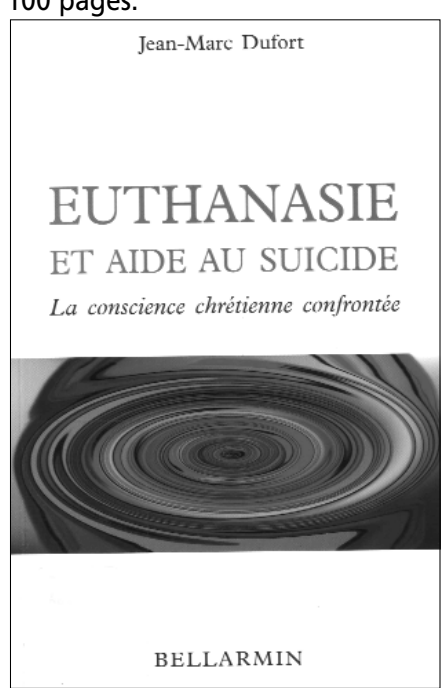

Jean-Marc Dufort, jésuite, a enseigné la théologie aux universités de Montréal et de Laval, ainsi qu'à I'UQTR. II participe aujourd'hui aux activités du service Albatros-04, ce qui l'amène à s'intéresser aux soins palliatifs. Son ouvrage est à la fois un examen des réalités inhérentes au débat de société sur l'euthanasie et l'aide au 
suicide. Sa réflexion, typiquement chrétienne, porte plus précisément sur le bien-mourir.

En introduction, l'auteur mentionne deux réalités qui apparaissent évidentes à toute personne qui observe ce débat de société depuis une vingtaine d'années: une part de plus en plus importante de la population canadienne est en faveur de l'euthanasie. L'ardeur du débat n'est pas sans provoquer un choc des idées en provenance des champs juridique, médical, éthique et religieux. L'auteur souligne que les interventions sont d'inégales valeurs; il relève la répétition de la formule "mourir dans la dignité», la réflexion approfondie sur la mort et le bien-mourir, les témoignages des personnes spécialisées dans les soins palliatifs. Avant d'amorcer son analyse, il définit I'euthanasie en ces termes: "le geste délibéré de provoquer la mort de quelqu'un par motif de compassion» (p. 9), qu'il distingue de l'euthanasie volontaire qui "consiste à faire mourir une personne par compassion à sa demande» (p. 10).

L'auteur aborde dans un premier chapitre trois cas récents de bioéthique ayant défrayé les manchettes des médias canadiens.

D'abord, celui de Nancy B.: une jeune Québécoise atteinte d'une maladie mortelle, complètement paralysée, maintenue en vie artificiellement au moyen d'un respirateur, n'ayant aucune qualité de vie et pas le moindre espoir de guérison. Elle songe à la possibilité qu'on lui retire tout soutien respiratoire. II ne s'agit pas ici d'un cas d'euthanasie, mais d'une mort naturelle par cessation de traitement.

En second lieu, le cas Sue Rodriguez, qui, lui, serait un suicide médicalement assisté. Cette résidente de la Colombie-Britannique est atteinte d'une maladie dégénérative incurable, la sclérose latérale amyotrophique. Elle n'est pas en phase terminale et sa souffrance peut être contrôlée. Son état physique est tel qu'elle doit avoir recours à une aide médicale pour mettre fin à ses jours. Consulté à ce sujet, un tribunal, s'appuyant sur le Code criminel canadien, exprime l'avis qu'il s'agirait d'un homicide. La parution de ce jugement provoque de nombreux commentaires dans tout le pays. Nombre d'arguments sont alors invoqués: droit de mourir dans la dignité, droit à la vie, droit à l'autodétermination de la personne, liberté de choix du patient, etc. Ce cas permet à la population canadienne de mieux connaître les enjeux inhérents à ces questions inédites.

L'auteur présente aussi le cas Robert Latimer. Un fermier de la Saskatchewan met à mort, en 1993, sa fille de 12 ans, une handicapée physique et intellectuelle. $\mathrm{Ce}$ cas pose beaucoup de questions puisqu'il ne s'apparente pas vraiment à de l'aide au suicide: la jeune fille est incapable de s'exprimer clairement sur son état, il s'agit d'une mort provoquée. Monsieur Latimer croit avoir agi par compassion.

Ces trois cas ont obligé les responsables politiques canadiens à prendre position sur des problèmes d'éthique et de droit plutôt controversés. Parmi les membres d'un Comité spécial du Sénat, il est apparu évident que les deux éléments essentiels de compassion et de pitié doivent être clairement définis afin de limiter les cas d'abus (p. 17), c'est-à-dire pour éviter d'utiliser les notions de compassion et de pitié de manière arbitraire avec, par exemple, des vieillards ou des personnes souffrant d'incapacités ou de handicaps. Le Comité spécial du Sénat refuse de décriminaliser l'«euthanasie involontaire», celle où le patient ne l'a ni voulue ni cherchée, car nul ne peut argumenter une telle décision sur la base de la compassion et de la pitié.

Les trois cas évoqués plus haut relèvent, d'après l'auteur, d'un même problème de société: la question du sens que les proches et le malade lui-même, et non pas seulement l'aidant, veulent conférer à un pareil geste. Or, selon lui, le fait de soulager la douleur par la mort constitue l'exacte négation de cette question. D'où l'intention poursuivie dans cet ouvrage: montrer, en invoquant diverses traditions, que la souffrance de la personne peut avoir un sens dans une société où il n'y a plus que "la mort en douce, assistée ou non, qui se présente à la conscience comme la seule issue valable» (p. 19)

Dans le second chapitre intitulé "L'évolution du monde juridique vers l'euthanasie», l'auteur dresse un bref portrait de la situation au Canada. Du côté de la Cour suprême, il existe un avis majoritaire selon lequel I'euthanasie et l'aide au suicide représentent des infractions criminelles. Cela étant dit, le fait que cet avis ait «laissé en suspens plusieurs questions a donné lieu à une recherche passionnée de consensus et à la manipulation de l'opinion publique» (p. 27). Cette manipulation proviendrait surtout, d'après l'auteur des groupes proeuthanasie qui avancent le plus souvent l'argument suivant: «le fait crée le droit». Enfin, il conviendrait, selon lui, que la législation à cet égard prévoie un jeu pouvant permettre de gérer les circonstances particulières, moralement compréhensibles et justifiables, histoire d'éviter l'un des effets les plus pervers de la conception moderne de I'idée de justice: réduire à néant le souci d'autrui.

Le chapitre suivant traite de la médicalisation de l'euthanasie. L'auteur évoque la croissance des pratiques euthanasiques dans certains milieux médicaux malgré les dispositions que le nouveau Code civil du Québec a énoncées en ce domaine. II dresse un bilan sommaire de la situation qui prévaut en Amérique du Nord. Reprenons quelques éléments de ce bilan. En Oregon, une loi «définit les conditions selon lesquelles le suicide assisté par un médecin sera considéré comme une pratique médicale ordinaire» (p. 34); le médecin Jack Kevorkian, qui a déjà aidé 27 personnes à se suicider depuis 1990 , est surnommé "Docteur suicide». En 1996, un tribunal de la Californie ouvre la voie juridique à la reconnaissance du suicide médicalement assisté. Au Québec, une infirmière est menacée de radiation par son Ordre pour avoir administré sans prescription de la morphine à une patiente souffrant d'un cancer en phase terminale. Dans ce bilan somme toute partiel, l'auteur perçoit une tendance vers la fondation d'une permission légale du suicide dans l'éthique médicale professionnelle. Selon lui, "on prétend faire passer la responsabilité de cet acte du domaine de la conscience morale à un jugement médical portant sur la mort comme thérapie» (p. 36). II s'attend à ce que l'on ait recours de plus en plus à l'euthanasie dans les années à venir en raison du vieillissement général de la population et des ressources financières limitées que les États peuvent consacrer à leur système de santé. Pour l'auteur, la décision d'une personne qui souffre se situe au-delà des considérations juridiques et thérapeutiques puisqu'il s'agit, d'abord et avant tout, d'une décision existentielle.

Le chapitre suivant est consacré précisément à ce qu'il conviendrait de privilégier au-delà de ces considérations: des soins palliatifs qui préservent la qualité de vie des patients de tout âge en phase terminale, «dispensés par un personnel infirmier et des accompagnants formés à cette fin et attentifs aux besoins des patients ainsi qu'à ceux de leurs familles» (p. 41). L'auteur défend le mouvement des soins palliatifs. II note que le mouvement des soins palliatifs a pris de l'ampleur à partir des années 1960 en Occident. II regroupe des soignants en provenance de diverses professions: médecins, infirmières, psychologues, personnel de la pastorale, etc. II vise à transformer la vie quotidienne de personnes de manière à limiter ce qui rend le recours à l'euthanasie comme I'unique option envisageable pour elles: la solitude, la crainte, la souffrance et l'angoisse.

Au Québec, des centres de soins palliatifs fonctionnent grâce aux dons généreux de la population et au dévouement de leurs bénévoles. Le principal défi à relever pour le soignant consiste en l'établissement d'un dialogue fécond avec le patient; quand la souffrance est partagée, elle devient davantage supportable. Ultimement, ce dialogue doit le mener à une redécouverte de luimême, de son entourage et à une acceptation pleine et entière de sa condition. Il pourra ainsi donner un sens à ses souffrances qui touchent différents niveaux de son identité: physique, moral et spirituel. Dans la perspective chrétienne qui est la sienne, l'auteur considère que "la souffrance est (au sens existentiel) un pur négatif par rapport à la tendance foncière au bonheur qui est inscrite au plus profond de notre être. (...) Elle contraint à rentrer en soi-même, à remonter, à partir d'une impuissance apparente et grâce à elle - jusqu'à la source même de sa vie» (p. 53).

L'avant-dernier chapitre de l'ouvrage expose le point de vue de l'éthique dans la perspective d'une instauration de soins palliatifs à l'ensemble des patients en phase terminale. Puisque, selon l'auteur, ces personnes cherchent bien davantage un changement de situation que l'euthanasie proprement dite, et qu'il est possible de juguler la douleur jusqu'à concurrence de $80 \%$ de son seuil, il convient de s'engager dans une révolution des soins palliatifs. Cette révolution devrait idéalement se réaliser à différents niveaux: communiquer aux médecins et aux infirmières les connaissances acquises en recherche et sur le terrain; favoriser l'accès du malade à un dialogue spirituel; susciter une parole entre le malade, la famille et les soignants; respecter et préserver la vie en lui donnant toutes ses «chances».

L'auteur examine deux principes en jeu dans le débat relatif à la légalisation de l'euthanasie: le 
principe d'autonomie, selon lequel "toute demande volontaire d'euthanasie est une manifestation de liberté qui n'a pas à être justifiée» (p. 66); et le principe de compassion, en raison de l'excès de souffrances qui rejaillit sur l'entourage du malade. II analyse brièvement deux catégories de patients: celle des personnes âgées, "un groupe humain particulièrement vulnérable à l'euthanasie» (p. 71); et celle des personnes atteintes du sida, qui constitue un cas à part en vertu de la publicité que les médias font à cette maladie et du cas aigu d'euthanasie que peut représenter le sidéen en phase terminale. L'auteur complète ce chapitre en donnant une définition de "la mort dans la dignité», qui lui est inspirée par David Roy, et en mentionnant quelques repères éthiques indispensables dans un contexte pluraliste: la présomption qui doit toujours rester en faveur de la vie, le respect de l'autonomie de la personne, la prise en compte effective de la qualité de la vie et de la protection des plus faibles.

Dans le dernier chapitre de son ouvrage, l'auteur dresse un bref inventaire des passages bibliques dans lesquels on retrouve les thèmes suivants: vie, vie éternelle, mort, suicide, souffrance et résurrection. Il cherche à trouver des éléments susceptibles de répondre convenablement à la double question incontournable: où allonsnous et pourquoi ? Plus particulièrement, il cherche à élaborer une réponse authentiquement chrétienne à la souffrance qui remet en cause le désir de vivre: "la souffrance apparaît comme condition de salut dans un monde de péché, et elle rapproche de Dieu ceux qui, comme Job, la reçoivent. Elle contraint l'homme à opter pour ou contre Dieu» (p. 83). De nombreux passages bibliques l'auteur puise ses propres certitudes: tant le suicide que l'euthanasie sont des idées qui vont à l'encontre du commandement formel de la Bible («Tu ne tueras point».); depuis la résurrection du Christ, la mort est vaincue grâce à Lui; croire en Lui, c'est reconnaître la signification nouvelle de la mort et de la vie humaines; la vie est un don gratuit de Dieu; le «bien-mourir» (eu-thanasie) «n'est ici pas autre chose que le sens (à la fois direction et signification) de la vie retournant à Celui qui l'a donnée» (p. 94).

L'ouvrage de Jean-Marc Dufort est accessible à un très large public. II est bien documenté, rédigé dans un style clair, mais il y manque parfois des références précises sur des documents cités. Bien qu'il s'agisse d'une réflexion typiquement chrétienne sur le bien-mourir, il contient une analyse fort éclairante des enjeux inhérents au débat public sur l'euthanasie et l'aide au suicide.

Bernard Jobin 